\title{
Improved Velocity Estimation Algorithm for Traffic Surveillance Radar Using Autofocus Technique
}

\author{
Yanhui Xu, Yong Li \\ College of Electronic and Information Engineering, Nanjing University of Aeronautics and Astronautics \\ CIST of NUAA \\ Nanjing, China \\ yanhui006@163.com
}

\begin{abstract}
This paper is about an interesting application of the Frequency Modulated Continuous Wave (FMCW) radar system for the traffic surveillance. By introducing a novel monitoring mode, it is designed to provide synchronously the velocity measurements of all the vehicles within the radar footprint. To achieve a precise estimation of the real speeds of the moving vehicles, a Joint Maximum Contrast and Map Drift (JMCMD) algorithm using the autofocus technique is proposed in this paper. Those autofocus techniques originated from synthetic aperture radar data processing are analyzed and coupled here to form a two-step algorithm. Firstly, the maximum contrast method is used for a coarse estimation to get the rough velocity of the target. Then, the more accurate velocity of the target is obtained by using the map drift algorithm. The simulation results illustrate the errors of the measurement accuracy are less than $1 \mathrm{~km} / \mathrm{h}$, which satisfy the practical requirements.
\end{abstract}

Index Terms-Frequency Modulated Continuous Wave (FMCW), Synthetic Aperture Radar (SAR), Parameter Estimation, Maximum Contrast, Map Drift.

\section{INTRODUCTION}

The automatic measurement of the velocities and lengths of the vehicles using radar techniques could help to realize the intelligent traffic control and improve the safety on the highways [1]. However, the conventional traffic surveillance radar systems suffer from both the high cost of its hardware and the corresponding large computation load due to the limitation of the individual measurement for a vehicle [2]. An interesting application of Frequency Modulated Continuous Wave (FMCW) radar systems for the traffic surveillance has been discussed in literatures [3]. The proposed FMCW radar system (see in Fig.1 (b)) has some properties that are distinctly different from the conventional one (see in Fig.1 (a)). It is obvious that the vehicles within the footprint of the radar beam can be illuminated simultaneously in this novel monitoring mode [4][5].

Most of the traffic microwave radars measure the speed by making use of the well-known Doppler effect [6]. In the conventional working geometry, it is essentially based on the measurement of radial velocities. However, in the proposed working mode, the radial component of the actual speed is insignificant because the moving direction of a vehicle is approximately perpendicular to the radar line-of-sight. In fact,

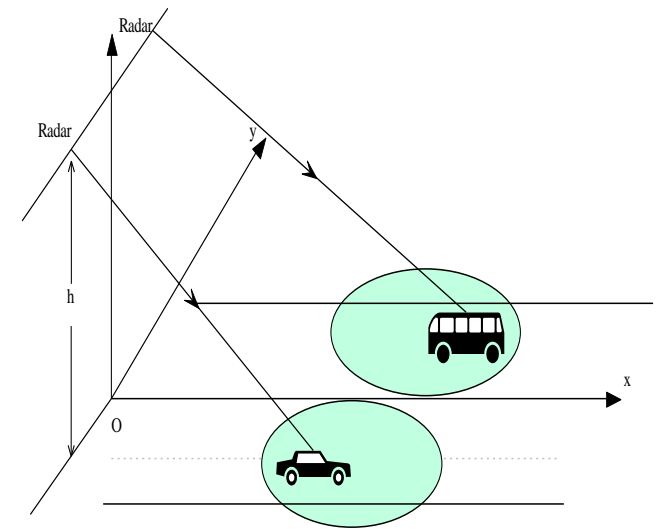

(a) Conventional Doppler radar

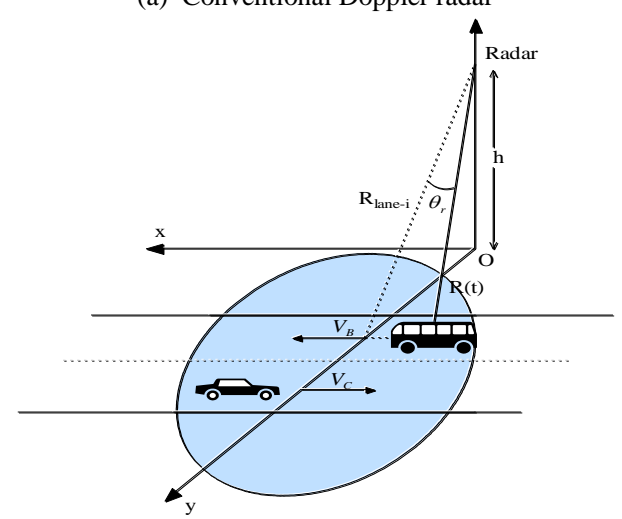

(b) Proposed FMCW radar

Fig.1. Two popular working geometry of traffic surveillance radar we present in this paper a robust method, i.e., a Joint Maximum Contrast and Map Drift (JMCMD) algorithm for estimating the tangential component of the moving vehicles.

\section{SIGNAL MODEL AND ANALYSIS OF SIGNAL CHARACTERISTICS}

The signal from the FMCW radar is [7]

$$
S_{t}(\tau)=w_{r}(\tau) \exp \left(j 2 \pi f_{0} \tau+j \pi K_{r} \tau^{2}\right)
$$

where $\tau$ is the time of the distance, $w_{r}(\tau)$ is the window function of the distance, $f_{0}$ is the carrier frequency and $K_{r}$ is the chirp rate.

The echo signal of the target B is 


$$
\begin{aligned}
S_{r}(\tau, t) & =A_{0} w_{r}(\tau-2 R(t) / c) \exp \left\{j 2 \pi f_{0}(\tau-2 R(t) / c)\right. \\
& \left.+j \pi K_{r}(\tau-2 R(t) / c)^{2}\right\}
\end{aligned}
$$

After the dechirp, the difference frequency signal is

$$
\begin{aligned}
s_{r}(\tau, t)= & A_{0} w_{r}(\tau-2 R(t) / c) \exp \left\{-j 4 \pi f_{0} R(t) / c\right\} \\
& \exp \left\{-j 4 \pi K_{r}\left(\tau-2 R_{r e f} / c\right)\left(R(t)-R_{r e f}\right) / c\right\}
\end{aligned}
$$

where $t$ is the time of the azimuth, $c$ is the speed of the light , $R(t)$ is the distance between the target B and the APC radar, $A_{0}$ is the amplitude, and $R_{r e f}$ is the reference distance. The $R(t)$ can be approximate as

$$
\begin{aligned}
& R(t)=\sqrt{R_{\text {lane } i}{ }^{2}+V_{B}^{2}\left(t-t_{c}\right)^{2}} \\
& \approx R_{\text {lane- } i}+\frac{V_{B}^{2}\left(t-t_{c}\right)^{2}}{2 R_{\text {lane- } i}}
\end{aligned}
$$

where $t_{c}$ is the time of the target B pass through the aperture center, $R_{\text {lane- } i}$ is the distance of the target B and the APC radar when the target $\mathrm{B}$ pass through the aperture center.

We define a oblique angle $\theta_{r}$ shown in Fig.1

$$
\theta_{r}=\arctan \left(-\frac{V_{B}\left(t-t_{c}\right)}{R_{\text {lane }-i}}\right)
$$

Then the Doppler center frequency of the echo signal $f_{d c}$ is

$$
\begin{aligned}
f_{d c} & =-\left.\frac{2}{\lambda} \frac{d R(t)}{d t}\right|_{t=t_{c}}=-\left.\frac{2 V_{B}^{2}\left(t-t_{c}\right)}{\lambda R_{\text {lane }-i}}\right|_{t=t_{c}} \\
& =+\left.\frac{2 V_{B} \sin \theta_{r}}{\lambda}\right|_{t=t_{c}}=\frac{2 V_{B} \sin \theta_{r c}}{\lambda}
\end{aligned}
$$

where $\lambda$ is the wavelength, $\theta_{r c}=\left.\theta_{r}\right|_{t=t_{c}}$ is the oblique angle when the target $\mathrm{B}$ pass through the aperture center. It is obvious that $\theta_{r c}$ is approximately zero, so $f_{d c}$ is also approximately zero, and $f_{d c}$ almost doesn't relate to the velocity. Thus, we can't get the velocity by estimating the Doppler center frequency.

Because the tangential velocity becomes higher when the radial velocity becomes lower, we can get another important Doppler parameter. We define $f_{d r}$ as the chirp rate of the echo signal

$$
\begin{aligned}
f_{d r} & =\left.\frac{2}{\lambda} \frac{d^{2} R(t)}{d t^{2}}\right|_{t=t_{c}}=\left.\frac{2 V_{B}^{2} \cos ^{2} \theta_{r}}{\lambda R_{\text {lane }-i}}\right|_{t=t_{c}} \\
& =\frac{2 V_{B}^{2} \cos ^{2} \theta_{r c}}{\lambda R_{\text {lane- } i}}
\end{aligned}
$$

In the equation (7), $\theta_{r c} \approx 0$, and $\cos \theta_{r c} \approx 1$, Thus

$$
f_{d r}=2 V_{B}^{2} / \lambda R_{\text {lane- } i}
$$

From equation (8), we can clearly see that the chirp rate only depends on the velocity. In another word, we can get the velocity by estimating the chirp rate. Then we will research the method to get an accurate chirp rate.

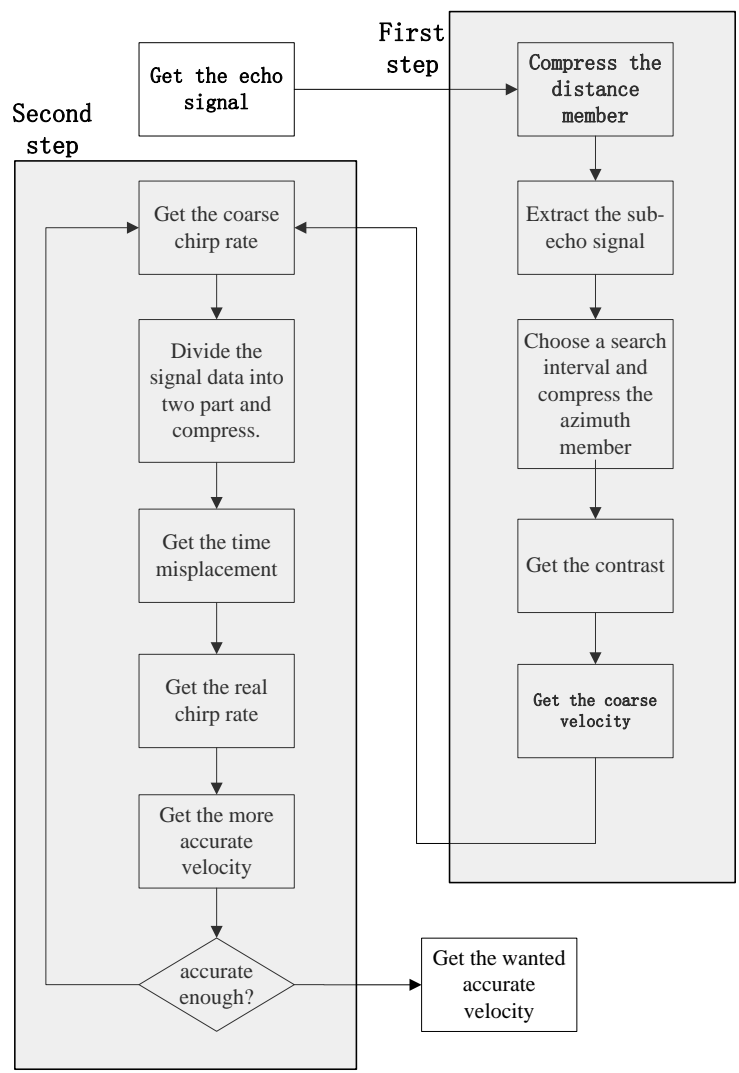

Fig.2. the flow chart of the proposed JMCMD method

\section{PROPOSED JMCMD METHOD}

Here we propose a Joint Maximum Contrast and Map Drift (JMCMD) algorithm to get an accurate velocity. The algorithm can be summarized as two steps. Firstly, the maximum contrast method is used for a coarse estimation to get the rough velocity of the target. Then, the more accurate velocity of the target is obtained by using the map drift algorithm. The flow chart of the method is shown in Fig.2.

We define a $M \times N$ radar images $\left\{s_{m, n}\right\}$, where $m$ is the azimuth number, and $n$ is the distance number. We also define

$$
\left\|S_{n}\right\|=\sum_{m=1}^{M} s_{m, n}{ }^{2},\left|S_{n}\right|=\left(\sum_{m=1}^{M} s_{m, n}\right)^{2} \text {, then we can get the }
$$

contrast of the radar images

$$
\text { Con }=\left\|S_{n}\right\| /\left|S_{n}\right|
$$

From the equation (9) and the meaning of the radar images, we can get that the contrast becomes bigger when the Doppler chirp rate is more accurate and the images focus better. Thus, when the contrast of the images is the maximum, the corresponding chirp rate is the real Doppler chirp rate. The maximum contrast method is summarized as follows:

(1) Compress the distance member of the echo signal

(2) Extract the sub-echo signal of each target by using the window function

(3) Choose a proper search interval according the priori information and use the search value to compress the azimuth member of the echo signal 
(4) Get the contrast of each sub-echo signal $X_{m}(n)$, which is compressed in the azimuth.

(5) Find the maximum of the contrast, and get the corresponding Doppler chirp rate and the coarse velocity.

The error of the azimuth chirp rate can cause the misplacement of the two compressed azimuth member. We can get the misplacement by measuring the processed data. This method called Map Drift uses the misplacement to get the Doppler parameter. The relationship between the misplacement time $\Delta \eta$ and the error of the azimuth chirp rate $\Delta f_{d r}$ is

$$
\Delta \eta=-\Delta f_{a}\left(\frac{1}{f_{d r}}-\frac{1}{f_{d r m f}}\right)
$$

where $f_{d r}$ is the real chirp rate, $f_{d r m f}$ is the coarse chirp rate estimated by the maximum contrast method, $\Delta f_{a}$ is the frequency interval of the center of the two data part, where data 1 represents the low part of the spectrum, and data 2 represents the high part of the spectrum.

From the equation (10), we can get that

$$
\begin{aligned}
\Delta f_{d r} & =-\frac{f_{d r m f}\left(f_{d r m f}-\Delta f_{d r}\right)}{\Delta f_{a}} \Delta \eta \\
& \approx-\frac{f_{d r m f}^{2}}{\Delta f_{a}} \Delta \eta
\end{aligned}
$$

Then we can get $f_{d r}$

$$
f_{d r}=f_{d r m f}-\Delta f_{d r}
$$

The algorithm of the JMMCD can be summarized as follows

(1) Get the coarse chirp rate $f_{d r m f}$ by the maximum contrast method, and divide the distance compressed echo signal data into two part.;

(2) Compress the two part data in the azimuth by using the coarse chirp rate $f_{d r m f}$, and get the time misplacement $\Delta \eta$;

(3) Get the error of the chirp rate $\Delta f_{a}$ according to the equation (11), and get the real chirp rate $f_{d r}$ according to the equation (12), and get the velocity according to the equation (8). Because of the rounding error and the inaccurate reading image, we shall do the algorithm Iteratively to get the more accurate velocity.

\section{SimUlations RESUlTS}

The parameters of the proposed FMCW radar system in the simulation are listed in Table 1. Two targets are shown in Fig.1 (b). Target $\mathrm{B}$ represents a slow-moving bus and target $\mathrm{C}$ is a high-speed car. They have opposite running directions according to the vehicle position in different lanes. It should be underlined that in Fig.3 we show four different velocity search intervals using the same maximum contrast algorithm in the range Doppler signal domain. As depicted in Fig.3, the optimal interval for measuring the target's coarse speed is $10 \mathrm{~km} / \mathrm{h}$. Fig.4 shows the two echo signal image compressed in the distance. And Fig. 5 shows the results of coarse velocity by the maximum contrast method. After the coarse search processing, the target's accurate velocities could be calculated using the map drift algorithm (see in Fig.6). The simulation results are shown in table 2. Following the proposed scheme of signal processing, we achieve a precise estimation which error is smaller than $1 \mathrm{~km} / \mathrm{h}$. The error is acceptable to maintain a relatively high accuracy and meet the requirement of the traffic surveillance system.

TABLE 1. Simulation Parameters

\begin{tabular}{|c|c|c|c|c|c|}
\hline \multirow[b]{2}{*}{ Target } & \multicolumn{3}{|c|}{ velocity $(\mathrm{km} / \mathrm{h})$} & \multicolumn{2}{|c|}{ Running Time (Sec) } \\
\hline & $\begin{array}{l}\text { Actual } \\
\text { Value }\end{array}$ & $\begin{array}{l}\text { Coarse } \\
\text { Value }\end{array}$ & $\begin{array}{c}\text { Accurate } \\
\text { Value }\end{array}$ & $\begin{array}{l}\text { Exhaustive } \\
\text { search } \\
\text { algorithm }\end{array}$ & $\begin{array}{l}\text { The } \\
\text { JMCMD } \\
\text { algorithm }\end{array}$ \\
\hline \multirow{2}{*}{$\begin{array}{c}\text { B } \\
\text { (Bus) }\end{array}$} & 18.0 & 20.0 & 18.3 & \multirow{2}{*}{1.875} & \multirow{2}{*}{0.266} \\
\hline & 42.0 & 40.0 & 41.8 & & \\
\hline \multirow{2}{*}{$\begin{array}{c}\mathrm{C} \\
(\mathrm{Car})\end{array}$} & 62.0 & 60.0 & 62.9 & \multirow{2}{*}{1.719} & \multirow{2}{*}{0.234} \\
\hline & 78.0 & 80.0 & 79.2 & & \\
\hline
\end{tabular}

\begin{tabular}{|c|c|}
\hline Center Frequency & $2 \mathrm{GHz}$ \\
\hline Radar Height & $15 \mathrm{~m}$ \\
\hline Signal Bandwidth & $80 \mathrm{MHz}$ \\
\hline Sampling Frequency & $4 \mathrm{MHz}$ \\
\hline Scene Extent & $15 \mathrm{~m} \times 15 \mathrm{~m}$ \\
\hline Squint Angle & Broadside \\
\hline Azimuth Integration Angle & $10^{\circ}$ \\
\hline Velocity Accuracy & $1 \mathrm{~km} / \mathrm{h}$ \\
\hline
\end{tabular}

TABLE 2. Velocity Estimation Results of Target B and C with the JMCMD Algorithm

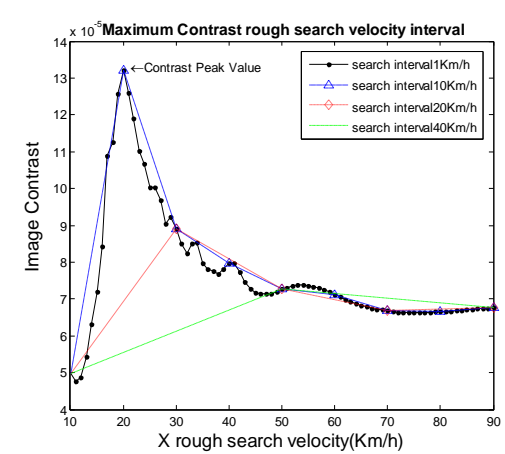

Fig.3. Demonstration of choosing different search interval in the coarse velocity estimation procedure

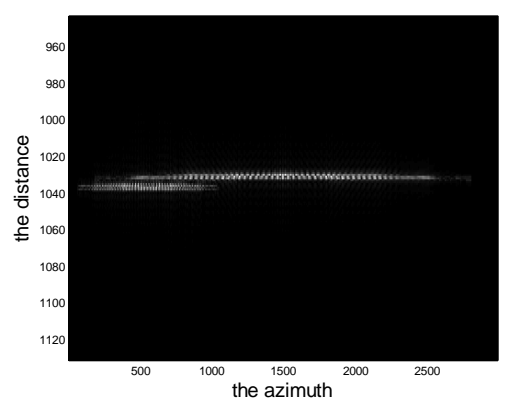

Fig.4. The two echo signal image compressed in the distance 


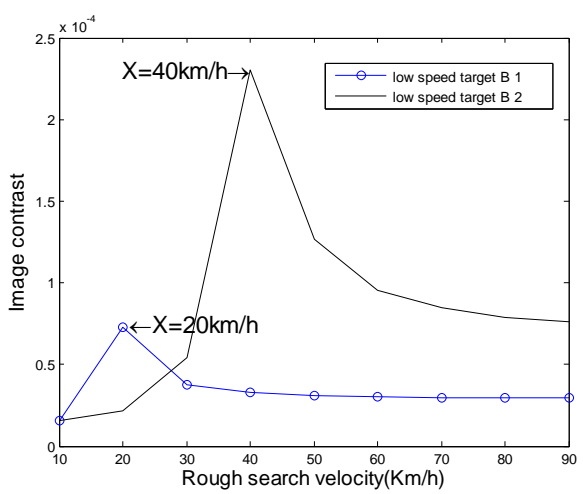

(a) Maximum contrast curve of target $B$

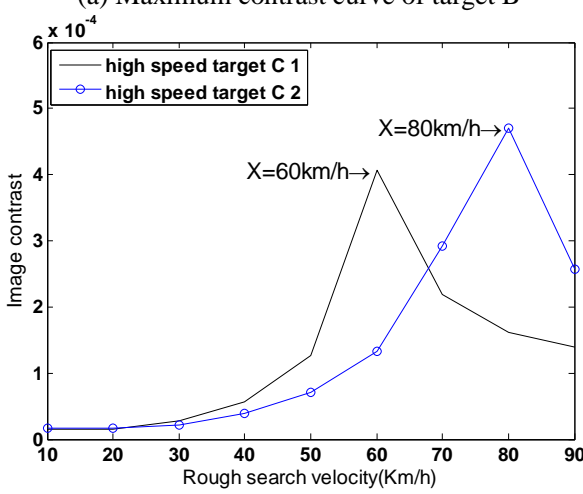

(b) Maximum contrast curve of target $\mathrm{C}$

Fig.5. Results of coarse velocity by the maximum contrast method

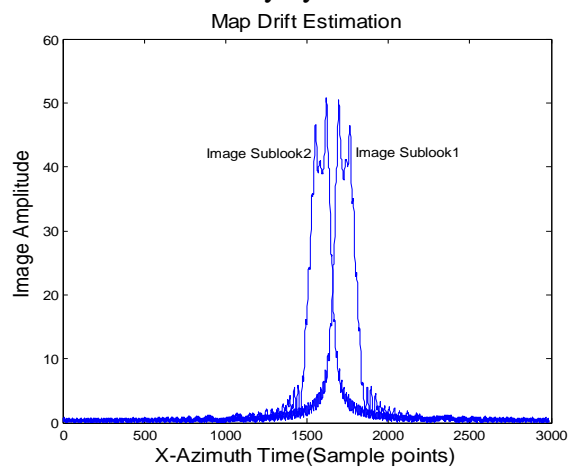

(a) map drift estimation of target $\mathrm{B}$

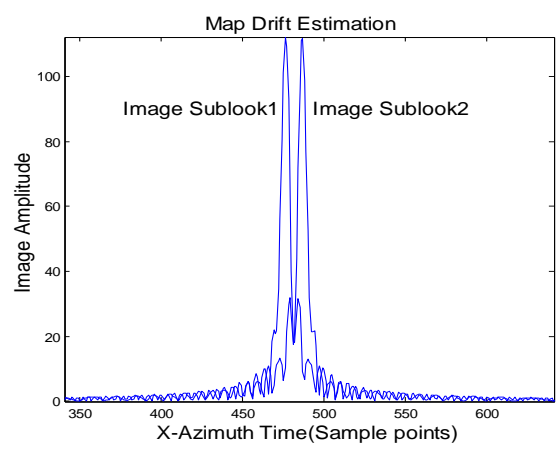

(b) map drift estimation of target $\mathrm{C}$

Fig.6. velocity estimation results of two targets with the JMCMD algorithm

\section{CONCLUSION}

Compared with the conventional Doppler radar system, the proposed FMCW radar system can provide real-time velocity values of the vehicles in multiple lanes of the traffic environment which is of great value for traffic surveillance applications. Through the introduction of the autofocus technique in SAR processing, the targets' velocity could be accurately estimated without heavy computation burden. The simulation results also illustrate the effectiveness of the proposed approach.

\section{REFERENCES}

[1] P. D. Fisher, "Improving on police radar", IEEE Spectrum, Vol. 29, No. 7, July 1992, pp. 38-43.

[2] Munoz-Ferreras, J.M., Perez-Martinez, F., Calvo-Gallego, J., et al. "A Traffic Surveillance System Based on a High Resolution Radar", IEEE Trans. on Geoscience and Remote Sensing, 2008, vol. 46, no. 6, pp.1624-1632I.

[3] Manyi Tao, Yong Li, "The Information Extraction Algorithm for a Traffic Detection Radar using ISAR principles", $20092^{\text {nd }}$ Asian-Pacific Conference on Synthetic Aperture Radar, Oct. 2009, Xi'an, P.R.China, pp.705-708.

[4] I.G. Cumming, Frank H. Wong, "Digital processing of Synthetic Aperture Radar data: Algorithms and Implementations", Artech House, 2005, pp.385-390.

[5] ANGEL Ribalta. "Time domain reconstruction algorithms for FMCW SAR", IEEE Geoscience And Remote Sensing Letters, 2011,8(3):396-400.

[6] JIANG Zhihong, HUANG Fukan, WAN J W.A, "chirp transform algorithm for processing squint mode FMCW SAR data". IEEE Geoscience and Remote Sensing Letters, 2007, 4(3):377-381.

[7] LIANG Yi, WANG Hongxian, XING Mengdao, et al. "The analysis of FMCW SAR signal and image study", Journal of Electronics \& Information Technology, 2008, 30(5): 1018-1021. 